\title{
Optimal management for people with severe spasticity
}

This article was published in the following Dove Press journal:

Degenerative Neurological and Neuromuscular Disease

3 October 2012

Number of times this article has been viewed

\author{
Jeffrey S Shilt ${ }^{1}$ \\ Pennie S Seibert ${ }^{2}$ \\ Vivek Kadyan' \\ 'Idaho Spasticity Program, Saint \\ Alphonsus Health Systems, Boise, ID, \\ ${ }^{2}$ Department of Psychology, Boise \\ State University, Boise, ID, USA
}

\begin{abstract}
Spasticity is characterized by velocity-dependent increase in tonic stretch reflexes and tendon jerks. Many people affected by spasticity receive late treatment, or no treatment, which greatly reduces the potential to regain full motor control and restore function. There is much to consider before determining treatment for people with spasticity. Treatment of pediatric patients increases the complexity, because of the substantial difference between adult and pediatric spasticity. Proper patient evaluation, utilization of scales and measures, and obtaining patient and caregiver history is vital in determining optimal spasticity treatment. Further, taking into consideration the limitations and desires of individuals serve as a guide to best management. We have grouped contributing factors into the IDAHO Criteria to elucidate a multidisciplinary approach, which considers a person's complete field of experience. This model is applied to goal setting, and recognizes the importance of a spasticity management team, comprising the treatment subject, his/her family, the environment, and a supportive, well-informed medical staff. The criteria take into account the complexity associated with diagnosing and treating spasticity, with the ultimate goal of improved function.
\end{abstract}

Keywords: spasticity treatment modalities, pediatric spasticity management, optimal management for spasticity

\section{Introduction}

Spasticity is clinically described as a motor disorder, characterized by a velocitydependent increase in tonic stretch reflexes and exaggerated tendon jerks, resulting from hyper-excitability of the stretch reflex, as one component of the upper motor neuron syndrome. Many diseases and injuries affecting the central nervous system (CNS) result in impairment of neuromuscular function, manifested as spasticity. Cerebral palsy (CP), multiple sclerosis (MS), traumatic brain injury (TBI), spinal cord injury (SCI), and cerebrovascular accident (CVA) are commonly encountered conditions that result in this neuromuscular dysfunction. In people with spasticity, neural impulses misfire, resulting in symptomatology that can affect both the upper and lower extremities.

An estimated 500,000 people in the United States, and twelve million people worldwide, experience spasticity. ${ }^{1}$ Yet, many of those affected are neglected, or treated late for their spasticity, and relegated to salvage treatment of end effects on the musculoskeletal system. This approach leaves little hope for regaining full motor control and restoring pre-disease or pre-injury function.

When treating spasticity, it is imperative to understand its functional effects, and recognize that spasticity is not always deemed detrimental by the affected person,
Correspondence: Pennie Seibert

1055 N Curtis Road, Boise,

ID 83706, USA

Tel +l 2083676750

Fax +I 2083676836

Email penseibert@msn.com 
or their caregiver. In its mildest form, spasticity may substitute for muscular weakness, aid in the preservation of muscle mass, and decrease the prevalence of deep venous thrombosis. ${ }^{2}$ However, spasticity is damaging in its more severe forms. Spasticity causes imbalanced muscle forces that act upon bones, joints, and the muscles themselves. This imbalance results in bony angular and torsional deformities, subluxation/dislocation of joints, capsular contractions, and muscle shortening. These findings were substantiated by Mercer Rang, who studied spastic mice that had developed restricted joint range, restrictive muscle excursion, and bony degeneration. ${ }^{3}$ These findings are considered to be the musculoskeletal ramifications of spasticity. Other skeletal ramifications include pain, casting or bracing challenges, skin complications, and challenges for fracture management. ${ }^{4}$ Additionally, spasticity impacts children differently than adults. Spasticity during growth compounds the soft tissue ramifications of increased tone. This is highlighted by excessive muscle shortening and abnormal bone growth, as a result of the imbalanced muscle forces acting upon growth plates. ${ }^{5,6}$

Many aspects are considered before deciding on treatment options for individuals with spasticity. These aspects not only include present-time quality of life, comfort, and function, but also future impairments, which may present as the result of growth, or expected decline in strength with age. Assessment of these aspects includes the patient's and the caregiver's perspectives. ${ }^{7}$

\section{Patient evaluation}

While it is easily recognized that severe TBI, CVA, and a variety of neurological disorders can result in spasticity, the actual diagnosis of spasticity is more complex. The complexity arises from challenges in distinguishing among abnormal patterns of muscle tone, such as volitional guarding, contracture, dystonia, paresis, paratonia, and rigidity. ${ }^{4,8-10}$ Furthermore, other movement disorders may be present in addition to spasticity. The incidence of these other movement disorders varies, depending upon the underlying etiology. It can be very frequent in cerebral palsy, rare following stroke (3.7\%), and nonexistent following spinal cord injury. ${ }^{11}$ Establishing the presence of accompanying movement abnormalities is important, as many of these are unaffected by traditional treatments for spasticity, and may appear more prominent when spasticity is reduced. Observation of the patient during tasks, and in ambulation, can identify these additional movement disorders by their salient features. ${ }^{12}$

Video analysis is extremely helpful in diagnosis, and serves as a useful aid in detecting change following treatment.
Though formal motion analysis in a gait lab is ideal, observational analysis with simple video is inexpensive, less labor- and time-intensive, and is often equally informative. We highly recommend obtaining video during an initial, thorough baseline physical therapy visit, when possible. Many picture archiving and communicating systems store these videos alongside radiographic studies, making them readily accessible.

During these initial sessions, passive range of motion is assessed during different velocities of movement to measure contracture and degree of spasticity. The Modified Ashworth Scale (MAS) is the most-utilized scale to measure spasticity. Since its inception, however, the validity and reliability of MAS has been challenged. To address issues with MAS, the Modified Modified Ashworth Scale (MMAS) was developed. In the revised scale, the ambiguity of the $1+$ grade has been omitted, and grade 2 has been redefined. MMAS ranges from 0-4: $0=$ no increase in muscle tone; $1=$ slight increase in muscle tone, manifested by a catch and release, or by minimal resistance at the end of the range of motion when the affected part(s) is moved in flexion or extension; 2 = marked increase in muscle tone, manifested by a catch in the middle range and resistance throughout the remainder of the range of motion, but the affected part(s) is easily moved; 3 = considerable increase in muscle tone, with passive movement being difficult; and $4=\operatorname{affected} \operatorname{part}(\mathrm{s})$ is rigid in flexion or extension. ${ }^{12}$ Though MMAS is not currently widely used, studies have supported the reliability of the scale. ${ }^{13}$ It allows grades to be more clearly defined, and provides for better clinical practice.

If appropriate, active motion is assessed for measures of function, timed functional trials, fatigability and repeatability, and selective motor control. A number of scales exist, which are used with the appropriate diagnoses and underlying impairment. ${ }^{14}$

Though physical examination is a critical component of patient evaluation, the patient and caregiver history is also integral to determining optimal treatment for spasticity. Understanding the limitations that each person experiences as a result of the spasticity, as well as understanding their respective desire for tone reduction and strengthening, helps guide best management. For example, with adults who rely upon stand-pivot transfers, and depend upon their spasticity to achieve this task, and who have either a low desire or an inability to strengthen adequately, intrathecal baclofen may not be the optimal choice to reduce spasticity. On the other hand, a very motivated young child, with substantial growth remaining, and a supportive family, may be an excellent 
candidate for intrathecal baclofen treatment, despite a possible temporary early reduction in function.

\section{Treatment options}

Two primary treatment algorithms exist for spasticity management, each dependent upon the magnitude of cognitive impairment. The ultimate treatment goal in the first group is to improve care and comfort for those who are significantly impaired, both physically and mentally, and who reply upon others for care. Functional improvement becomes increasingly challenging as cognitive ability declines, while improving comfort greatly improves quality of life for both the impacted individual and their caregiver. Most families and caregivers are satisfied with improved comfort, increased quality of life, and eased caregiving as outcomes of this treatment. The benefits of such an approach are widely documented in the literature. ${ }^{15}$

For the population who are physically debilitated but who have lesser degrees of mental impairment, the roles of spasticity reduction become less clear. The controversies include:

1. Weighing spasticity reduction against potential loss of function

2. Spasticity reduction without an associated improvement in function.

A common belief is that spasticity reduction options that impact on function should be avoided. This phenomenon is commonly seen in young individuals with spastic diplegic cerebral palsy, and in adult stroke or incomplete spinal cordinjured patients who live independently. For example, caregivers and family members who witness an initial loss of ambulation or transfer ability following an intrathecal baclofen trial will often deem it a failure. Though this snapshot of function may be accurate, ignoring the implications of long-term spasticity may be nearsighted. Though spasticity may aid in short term preservation of function, it commonly results in long-term musculoskeletal impairments, such as joint contractures, muscle shortening, and bony deformity. Oftentimes, pediatric patients who substitute for weakness with spasticity will ultimately lose any function gained from utilizing their increased tone, as their mass increases. ${ }^{16}$ Therefore, it is important to consider the long-term implications of spasticity, and to weigh it against short-term gains, to avoid this outcome.

Another controversial issue centers on the prevailing historical view that spasticity management should lead to measurable and immediate improved function in order to be considered an effective treatment. Though it is clearly a desirable outcome, there are many other elements that affect function, making this short- to mid-term goal impossible to assess. Currently-used scales perform well in measuring different abilities directly or indirectly impacted by increased tone, but do not address the aforementioned aspects, which contribute to functional goal attainment, alongside inherent ability. For example, the Gross Motor Function Classification System (GMFCS) is an excellent classification of mobility and ambulatory ability. The GMFCS and similar scales simply describe categories in which the individual resides. ${ }^{17}$ As a result, these scales limit assessment of potential through not taking into account individuals' characteristics and their environmental support.

In response to the limitations of predicting outcomes using current classification systems, assessment tools, and scales, we devised a concise collection of a small number of variables, which we have found to influence function in individuals experiencing spasticity, and developed the IDAHO Criteria (Figure 1). It is similar in concept to the World Health Organization's International Classification of Functioning, Disability and Health (ICF), ${ }^{18}$ which is formidable and rarely used clinically or otherwise. ${ }^{19}$ The ICF is exhaustively detailed in compiling factors to measure health and disability, and serves as a valuable research tool. The IDAHO Criteria offers a simple alternative schematic to assist in evaluating an individual's potential. This model guides goal setting, and recognizes the importance of many factors integral to attaining functional improvement; treatment options are evaluated holistically for optimal outcomes. Approaches that consider the processes involved with health care delivery have demonstrated improved patient and family satisfaction..$^{20,21}$

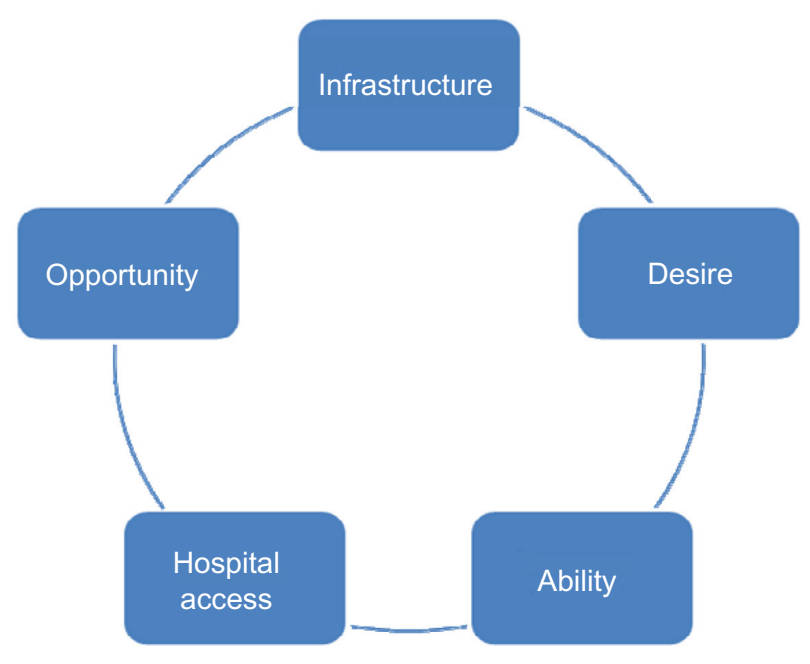

Figure I The IDAHO criteria. 
First, we assess the individual's Infrastructure (I), which is the supportive environment in which they live. Spastic individuals who have full-time emotional and physical support have a distinct advantage. Access to essential exercise equipment and the opportunity to learn proper musclestrengthening techniques are optimal for improving muscle strength and subsequent function.

Next, we assess the individual's Desire (D), which pertains to their motivation and courage in generation and pursuit of stated outcomes. Desire is an especially vital component, which must be properly addressed in order to confirm that the person, his/her family members, and medical staff are all fully aware of the person's situation, thereby permitting open communication and establishing realistic goals. It is imperative to define Desire because, frequently, the person and his/her family members, prior to consultation with the physician, may set unreasonable goals regarding spasticity management; when these exalted goals are not achieved as imagined, discouragement may develop, and impede the recovery progress. ${ }^{4}$ However, through effective communication and establishing practical, realistic goals, improvement can be facilitated. Appropriately addressing the Desire component can help to avoid discouragement, and regression in recovery can be avoided.

Ability (A) refers to an individual's inherent physical and mental characteristics. This is based on both the level of impairment and the pre-existing physical state. For example, irrespective of the severity of spasticity, a person with cognitive impairment cannot be assumed to have the same ability to improve as a person without deficits. Additionally, selective motor control greatly enhances one's ability to strengthen, following spasticity reduction.

Hospital Access $(\mathrm{H})$ is defined by proximity of the subject's residence to professional care services. Hospital access demonstrates the existence of geographic disparities, in which location is a leading determinant in whether or not health care is accessible, along with the ease of access. Statistics indicate that, in the United States, a quarter of Hispanics, and a third of African-Americans and American Indian/Alaskan natives live outside metropolitan areas where suitable hospitals are located. Consequentially, a large proportion of people do not receive adequate attention and appropriate care, solely due to their location. ${ }^{22}$ Similarly, in Third World countries, access to state-of-the-art care is limited. The Hospital Access component of the criteria encourages the recognition of location, and the importance of understanding that location has a significant impact, not only on the person's treatment plans but also on proper diagnosis.
Finally, Opportunity (O) signifies the economic aspect of access to specialty care and treatment opportunities, which has the potential to drastically change the treatment directive. ${ }^{23}$ Research has revealed that ethnic and racial minorities are statistically shown to have incomes that fall below 200 percent of the federal poverty level, suggesting that they are more likely to be uninsured, in comparison to other Americans. Undoubtedly, this implies that a tremendous disparity in health care delivery is associated with socioeconomic status, which must be recognized when performing evaluations. ${ }^{22}$

Unfortunately, because of health care disparities, those affected by socioeconomic and geographic disparities are also affected by disparities in opportunity. Based on their lowincome status, some people are unable to access specialized physicians at high-quality hospitals. For this reason, their goals and their treatment routes may not be suitable. Thus, proper improvement may not be achieved. ${ }^{22}$

Treatment options are based on the determination of localized or generalized spasticity, its level of severity, degree of selective motor control, cognitive function, and the personal needs of the individual. Once these factors have been analyzed, different modalities of treatment are considered, including nonpharmaceutical, oral medication, intramuscular injection, and intrathecal medication.

Nonpharmaceutical methods, which have been utilized for many decades to manage spasticity, include bracing, casting, occupational therapy, physiotherapy, and surgery. Although these methods fail to eliminate the underlying issue, they do provide support, substitute for weak muscles, and maintain a range of joint motion, in order to alleviate the challenges of daily activity. ${ }^{24}$ While nonpharmaceutical and nonsurgical options may aid individuals in mobility, the extent to which they are considered successful in improving range of motion remains debated. Soft tissue surgical interventions are utilized as well. But surgery does not directly address spasticity. Surgery should be considered a salvage method, to optimize outcomes once spasticity has been adequately treated. This is particularly true in the pediatric population, wherein repeated lengthening of the musculotendinous unit in a growing child often results in weakening of muscle. Furthermore, it is the experience of the author that outcomes achieved by surgery, regardless of the age when performed, are best maintained by optimal spasticity management. Selective dorsal rhizotomy (SDR) is another surgical option for spasticity management. Historically, it has been reserved for children with spastic diplegia who have very good strength. A few studies have demonstrated good long-term outcomes with this method. The challenge in 
this conclusion is that children with a degree of underlying ability do well regardless of the management chosen. ${ }^{25-27} \mathrm{In}$ addition, this surgery is irreversible. Spasticity often returns with maturity, and secondary deformities occur. ${ }^{28}$

Traditionally, orally-administered medications have been the primary and most widely used method of treatment for spasticity. Some commonly used oral medications include gamma amino butyric acid (GABA) agonists (such as baclofen and benzodiazepines) and muscle relaxants (such as dantrolene and $\alpha 2$-adrenergic agonists, including tizanidine). ${ }^{4,24}$ Unfortunately, these medications permit shortterm benefits but frequently lead to further complications. In cases of severe spasticity, oral medications reduce tone inadequately; the tone reduction achieved fluctuates and does not provide a steady state of effect. ${ }^{4}$

Furthermore, the sedating effects of the oral baclofen dosages required to provide adequate spasticity management commonly interfere with cognitive function and development. ${ }^{29}$ In addition to drowsiness, the systemic side effects of dantrolene and tizanidine must be closely monitored, to avoid hematologic and hepatic damage.

Investigations of focal spasticity have shown that localized interventions are a more successful method of treatment. Intramuscular injections, which include injections of botulinum toxin, alcohol, and phenol have shown to be more effective in directly treating the spastic muscle and decreasing adverse side effects. ${ }^{4}$

Botulinum toxin is a natural toxin formed from Clostridium botlinum bacteria. It acts by inhibiting the release of acetylcholine at the neuromuscular junction, with high specificity, and has been demonstrated to be safe and efficacious in treating abnormally increased muscle tone..$^{30,31}$ Botulinum toxin takes effect two to three days after injection, and peaks clinically at 4-6 weeks, gradually wearing off at 3-4 months. This leads to staggered results. Those who receive the maximum number of injections are relieved, for various episodes, approximately 4 months out of a year. ${ }^{32}$ However, in patients with good selective motor control, the positive results can persist for 6 months or longer. Individuals with selective motor control of the antagonistic muscles are ideal candidates for botulinum toxin injections.

Alcohol and phenol are both nonselective chemodenervating therapeutic agents, and are less diffusive than botulinum toxin. They are less costly options, and are advantageous in their rapid onset and localized potency, but pose possible risks of soft tissue fibrosis development, as well as dysesthesia. ${ }^{33}$ Also, alcohol and phenol are difficult to inject, requiring perineural placement, or placement near the point of motor innervation. ${ }^{23}$ The effects of phenol are irreversible, and may reduce contraction during voluntary movements, produce scarring, granuloma formation, and edema. ${ }^{24}$ Additionally, given the shorter duration of effect of alcohol injections, they are less commonly employed.

Finally, intrathecal baclofen (ITB), approved by the Food and Drug Administration in 1996, is being used to treat spasticity. Instead of paralyzing muscle cells, ITB works as a GABA agonist. GABA is an inhibitory neurotransmitter, which relaxes antagonist muscles (ie, reduces co-contraction). Baclofen acts to stimulate GABA receptors, directly effecting muscle tone and spasticity. Oral baclofen crosses the blood-brain barrier poorly, preventing its effective entrance into the CNS. When delivered intrathecally, its cerebrospinal fluid concentrations are a hundredfold greater than those obtained by oral ingestion. Intrathecal baclofen has fewer systemic side effects compared to oral baclofen. However, the procedure does include additional surgical risks and possible complications. ${ }^{34}$ Evidence supports the efficacy of ITB, and indicates greatest efficacy in people with severe spasticity. ITB helps to reduce pain, improve sleep, improve daily living activities, improve abilities, and enhance quality of life. ${ }^{15}$ ITB is now a commonly practiced therapy, which reduces spasticity in people who have $\mathrm{CP}$, and has been proven effective by multiple studies. ${ }^{35}$

\section{Treatment practices}

After agreeing on treatment goals with the person and caregivers involved, the use of our treatment algorithm is based upon the degree of body involvement and the cognitive level of the person.

Persons with spasticity limited primarily to muscle groups in one limb are excellent candidates for neuromuscular blockade with botulinum toxin, phenol or a combination of the two. In patients with selective motor control, we are more aggressive, with repeat injections accompanied by physical therapy, bracing, electrical stimulation, and other supportive modalities. Injections should be continued on a regular basis at three to four times per year. ${ }^{33}$ Unsuccessful trials should lead to pursuing treatments similar to those for generalized spasticity. People commonly encountered in this group include individuals afflicted by stroke and hemiplegic or diplegic cerebral palsy. Neuromuscular blockade can be utilized as an adjunctive therapy, alongside intrathecal baclofen therapy, SDR, and musculoskeletal surgery.

Treating generalized spasticity is more complex. Oral medication is commonly used as an initial form of treatment. However, due to commonly encountered sedation, this can be 
counterproductive for participation in physical, occupational, and speech therapies, as well as for cognitive development. For people with severe impairments, demonstrating an absence of cognitive development, the level of sedation that often accompanies oral medications may be acceptable and/or desirable. This scenario is commonly encountered in spastic quadriparetic CP and TBI. Care must be exercised following TBI, as sedation may mask cognitive recovery.

ITB therapy is considered in individuals with generalized spasticity, with intact or developing cognition, and/or who fail oral medications. ${ }^{36}$ While the use of ITB therapy to treat spasticity is becoming more commonly practiced, it is often overlooked. ITB therapy has been the most universally studied option for the treatment of spasticity related to CP. Less research has been done to indicate ITB's significance for people with other underlying injuries and diseases of the CNS, such as CVA, TBI, MS, and SCI. For example, an estimated $17 \%-42.6 \%$ of CVA survivors experience spasticity, but less than $1 \%$ of those who experience a disabling form of spasticity receive ITB treatment. ${ }^{37}$ The original labeling of baclofen suggested waiting one year after SCI, and 6 months following CVA. Our anecdotal experience is that earlier treatment may avoid many of the musculoskeletal complications of spasticity. This has been demonstrated (in the orthopedic literature) in patients with cerebral palsy. ${ }^{38}$ Ongoing trials are underway to help clarify this issue. This therapy can be considered in growing, and adult, patients with di-, hemi- or quadriparetic CP, TBI, MS, or SCI, regardless of cognitive status.

Nonpharmacological treatments are important adjuncts when treating spasticity, whether it is generalized or focal (Figure 2). Nonpharmacological approaches include occupational therapy, physiotherapy, orthotics, casting, or a combination of these methods. Strength training is imperative to help restore muscle balance. Muscle balance allows normal muscle, bone, and joint development and maintenance. Achieving strength is a key component in reaching normal function. There are many variables responsible for restoring muscle balance beyond the physiologic ability of muscle to be strengthened. This is one of the more critical areas where the IDAHO criteria can be useful in assessing the optimal delivery of care.

\section{Pediatric considerations}

While CVA is the most common cause of spasticity in adults, the most common causes in children are CP, TBI, and spinal cord injury. ${ }^{39}$ There is a substantial difference in the impact of spasticity on children, versus adults. In adults who experience

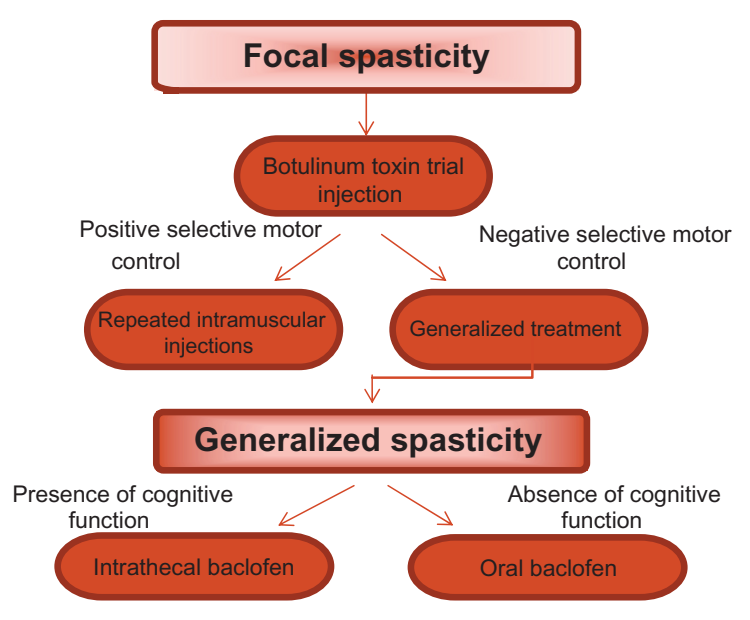

Figure 2 Treatment practice in focal and generalized spasticity.

spasticity, inherent muscle length is present, and there is no deficiency in normal muscle length and architecture. The effects of spasticity in children can be substantially more devastating, due to the effects of muscle tightness on the growing musculoskeletal system. Unlike bone, muscle does not have a growth plate. Muscle growth is directed by the tensile forces of the bone, pulling under physiological loading as the bone elongates, which subsequently causes relaxed muscles to stretch. ${ }^{28}$ Therefore, muscle grows at the rate of bone growth. Children with $\mathrm{CP}$ are musculoskeletally normal at birth, with spasticity occurring during rapid growth, preventing normal bone and muscle development. ${ }^{40}$ This muscle shortening can be substantial. In a comparative study, longitudinal muscle growth was reduced by $45 \%$ in spastic mice, compared with normal mice. ${ }^{41}$ This restricted longitudinal muscle growth and increased tension of the muscle can result in limb deformities, joint dislocations, and poor motor function..$^{42}$

There are many factors to consider when treating children with spasticity. A study by Gorter et al found that spasticity is only marginally related to gross motor function development in infants of less than 2 years of age who have CP, and that other environmental or family factors may have a much greater influence on gross motor function. ${ }^{42-44}$ Here, again, it is important to account for factors other than direct interventions, in considerations of functional gains. We know that severe spasticity often inhibits motor activity, and that treating this spasticity may promote development of motor pathways, by allowing more movements. ${ }^{39}$ By aggressively treating spasticity early, we may be preserving opportunity for future gains.

Despite considerable costs, treating spasticity in children has shown to be both cost-effective and beneficial. ${ }^{45}$ 
A study performed by Kan et al compared preoperative and postoperative data, using MAS and GMFCS, for two groups of children who received either ITB pumps or SDR. The results 1 year post-treatment were decreased tone, increased range of motion, and improved function, for both ITB and SDR groups, showing both surgical treatments to be efficacious for children with moderate to severe spasticity. The benefits of these successful treatments continue. There was a significant decrease in the number of subsequent orthopedic procedures needed, and a parents' satisfaction rate of $95.8 \%$ with these treatments. ${ }^{1}$ Having an improved range of motion and function not only improves quality of life for the child, but also eases the burden of the parents in providing proper care.

It has been observed that early treatment of spasticity reduces the need for orthopedic surgery for contracture or torsion deformity in children with severe spasticity from CP. ${ }^{42,45}$ Eliminating tone allows children to strengthen and achieve motor control, thereby preventing the musculoskeletal ramifications of spasticity. Evaluating functional improvement can be challenging. Though a child may be improving, he or she may also be moving away from the normal trajectory. For example, children with diplegic $\mathrm{CP}$ generally experience improvements until they reach age six, at which point function often plateaus or declines. Up until this time, spasticity can substitute for underlying weakness. However, as they mature skeletally and gain weight, spasticity becomes a poor surrogate for normal muscular balance and strength. This can be explained by a power (strength) to weight ratio; young children have a high power to weight ratio. However, as children age, their mass begins to increase more rapidly in proportion to their physical strength, resulting in a reversal: to a low power to weight ratio. We must be diligent in avoiding the preservation of spasticity for this deceptive "function," while it impairs normal musculoskeletal development.

\section{Conclusion}

It is well-established that oral, intramuscular, and intrathecal medications are effective in decreasing spasticity. The management of severe spasticity in order to improve function, however, involves a complex web of intervening variables, including infrastructure, desire and motivation to make lifestyle changes, ability remaining following injury, hospital access to appropriate care, and opportunity. Spasticity is the critical impediment that must be removed for individuals striving to reach higher function, or to improve the care and comfort of those with severe cognitive impairment.

\section{Acknowledgments}

We would like to acknowledge Julie Schommer, Jennifer Valerio, and Yustina Rafla of the Department of Psychology, Boise State University for their contributions to the preparation of this manuscript. There was no financial or material support given.

\section{Disclosure}

The authors report no conflicts of interest in this work.

\section{References}

1. Kan P, Gooch J, Amini A, et al. Surgical treatment of spasticity in children: comparison of selective dorsal rhizotomy and intrathecal baclofen pump implantation. Childs Nerv Sys. 2007;24:239-243.

2. Bakheit AMO. Botulinum Toxin Treatment of Muscle Spasticity. Milton Keynes, UK: AuthorHouse; 2007.

3. Rang M. Cerebral palsy. In: Morrissy R, editor. Lovell and Winter's Pediatric Orthopedics. Philadelphia: Lippincott; 1990;3: 465-506.

4. Green NE, Swointkowski MF, editors. Skeletal Trauma in Children, 4th ed. Philadelphia, PA: Saunders, Elsevier; 2008.

5. Gage J. Managing spasticity in children with cerebral palsy requires a team approach. A Pediatric Perspective. 2004;13(3):1-6.

6. Goldwyn A, Pountney T. Theoretical aspects of postural management Proceedings of the 19th International Seating Symposium; February 27-March 1, 2003; Orlando, FL.

7. Charles PD, Gill CE, Taylor HM, et al. Spasticity treatment facilitates direct care delivery for adults with profound intellectual disability. Mov Disord. 2010;25(4):466-473.

8. Dietz V, Sinkjaer T. Spastic movement disorder: impaired reflex function and altered muscle mechanics. Lancet Neurol. 2007;6:725.

9. Pauca R, Young A. Paratonia and gegenhalten in childhood and senescence. Clin Chiropr. 2012;15(1):31-34.

10. Hobbelen JS, Koopmans RT, Verhey FR, Van Peppen RP, de Bie RA. Paratonia: a Delphi procedure for consensus definition. J Geriatr Phys Ther. 2006;29(2):50-56.

11. Handley A, Medcalf P, Hellier K, Dutta D. Movement disorders after stroke. Age Ageing. 2009;38(3):260-266.

12. Abdo WF, Van de Warrenburg BPC, Burn DJ, Quinn NP, Bloem BR. The clinical approach to movement disorders. Nat Rev Neurol. 2010;6:29-37.

13. Ghotbi N, Ansari NN, Naghdi S, Hasson S. Measurement of lower-limb muscle spasticity: intrarater reliability of modified modified Ashworth scale. J Rehabil Res Dev. 2011;48(1):83-88.

14. Yelnik AP, Simon O, Parratte B, Gracies JM. How to clinically assess and treat muscle overactivity in spastic paresis. J Rehabil Med. 2010;42:801-807.

15. Dan B, Motta F, Vles JSH, et al. Consensus on the appropriate use of intrathecal baclofen (ITB) therapy in paediatric spasticity. Eur J Paediatr Neurol. 2010;14(1):19-28.

16. Kim WH, Park EY. Causal relation between spasticity, strength, gross motor function, and functional outcome in children with cerebral palsy: a path analysis. Dev Med Child Neurol. 2011;53:68-73.

17. Morris C. Current and future uses of the gross motor function classification system: the need to take account of other factors to explain functional outcomes. Dev Med Child Neurol. 2010;51(12):1003.

18. World Health Organization. International classification of functioning, disability and health. Available from: http://www.who.int/ classifications/icf/en. Accessed March 21, 2012.

19. Ptyushkin P, Vidmar G, Burger H, Marincek C. Use of the international classification of functioning, disability, and health in traumatic brain injury rehabilitation: linking issues and general perspectives. Am J Phys Med Rehabil. 2012;91(13):S48-S54. 
20. Woodside JM, Rosenbaum PL, King SM, King GA. Family-centered service: developing and validating a self-assessment tool for pediatric service providers. Child Health Care. 2001;30:237-252.

21. Rosenbaum P. Family and quality of life: key elements in intervention in children with cerebral palsy. Dev Med Child Neurol. 2011;53:68-70.

22. First W. Overcoming disparities in US health care. Health Affairs. $2005 ; 24: 445-451$

23. Shilt J, Reeves S, Lai L, et al. The outcome of intrathecal baclofen treatment on spastic diplegia: preliminary results with a minimum of two year follow-up. J Pediatr Rehabil Med. 2008;1(3):255-261.

24. Koman A, Smith B, Shilt J. Cerebral Palsy. Lancet. 2004;363: 1619-1631.

25. Farmer J, Sabbagh S. Selective dorsal rhizotomies in the treatment of spasticity related to cerebral palsy. Childs Nerv Sys. 2007;23: 991-1002.

26. Nordmark E, Josenby AL, Lagergren J, Andersson G, Strömblad L, Westbom I. Long-term outcomes five years after selective dorsal rhizotomy. BMC Pediatr. 2008;8:54.

27. Langerak N, Lamberts R, Fieggen A, Peter J, Peacock W, Vaughan C. Selective dorsal rhizotomy: long-term experience from Cape Town. Childs Nerv Sys. 2007;23:1003-1006.

28. Farmer J, Sabbagh AJ. Selective dorsal rhizotomies in the treatment of spasticity related to cerebral palsy. Childs Nerv Sys. 2007;23: 991-1002.

29. Dario A, Tomei G. A benefit-risk assessment of baclofen in severe spinal spasticity. Drug Saf. 2004;27(11):799-818.

30. Schulte-Mattler W. Use of Botulinum toxin A in adult neurological disorders: efficacy, tolerability, and safety. CNS Drugs. 2008;22(9):725-738.

31. Kadyan V, Clairmont AC, Engle M, Colachis SC. Severe trismus as a complication of cerebrovascular accident: a case report. Arch Phys Med Rehabil. 2005;86(3):594-595.

32. Pathak MS, Nguyen HT, Graham HK, Moore AP. Management of spasticity in adults: practical application of botulinum toxin for the treatment of wrist and finger spasticity after a stroke. Eur J Neurol. 2006;13:42-50.
33. Elovic E, Esquenazi A, Alter K, Kin J, Alfaro A, Kaelin D. Chemodenervation and nerve blocks in the diagnosis and management of spasticity. Am Acad Phys Med Rehabil. 2009;1:842-851.

34. Russman B. Intrathecal baclofen. Dev Med Child Neurol. 2010; 52(7):601

35. Shilt J, Lai L, Cabrera M, Frino J, Smith B. The impact of intrathecal baclofen on the natural history of scoliosis in cerebral palsy. J Pediatr Orthop. 2008;28:684-687.

36. Dario A, Tomei G. A benefit-risk assessment of baclofen in severe spinal spasticity. Drug Saf. 2004;27(11):799-818.

37. Dvorak E, Ketchum N, McGuire J. The underutilization of intrathecal baclofen in poststroke spasticity. Top Stroke Rehabil. 2011;18(3):195-202.

38. Krach LE, Kriel RL, Gilmartin RC, et al. Hip status in cerebral palsy after one year of continuous intrathecal baclofen infusion. Pediatr Neurol. 2003;30(3):163-168.

39. Centers for Disease Control and Prevention. Stroke facts and statistics. Available from: http://www.cdc.gov/stroke/stroke_facts.htm. Accessed July 7, 2011.

40. Graham HK, Selber P. Musculoskeletal aspects of cerebral palsy. J Bone Joint Surg Am. 2003;85-B:157-166.

41. Ziv I, Blackburn N, Rang M, et al. Muscle growth in normal and spastic mice. Dev Med Child Neurol. 1984;26:94-99.

42. Bakheit A. The use of botulinum toxin for the treatment of muscle spasticity in the first 2 years of life. Int J Rehabil Res. 2010;33:104-108.

43. Gorter JW, Verschuren O, van Riel L, Ketelaar M. The relationship between spasticity in young children (18 months of age) with cerebral palsy and their gross motor function development. BMC Musculoskelet Disord. 2009;10:108.

44. Balkrishnan R, Camacho FT, Smith BP, et al. Cost impact of botulinum toxin therapy in medicaid-enrolled children with cerebral palsy. J South Orthop Assoc. 2002;2:71-82.

45. Gersten PC, Albright AL, Johnstone GF. Intrathecal baclofen infusion and subsequent orthopedic surgery in patients with spastic cerebral palsy. J Neurosurg. 1998;88:1009-1013.
Degenerative Neurological and Neuromuscular Disease

\section{Publish your work in this journal}

Degenerative Neurological and Neuromuscular Disease is an international, peer-reviewed, open access journal focusing on research into degenerative neurological and neuromuscular disease, identification of therapeutic targets and the optimal use of preventative and integrated treatment interventions to achieve improved outcomes, enhanced

\section{Dovepress}

survival and quality of life for the patient. The manuscript management system is completely online and includes a very quick and fair peer-review system. Visit http://www.dovepress.com/testimonials.php to read real quotes from published authors. 